experienced endoscopists thereby enabling the full potential of this novel imaging technology to be realised.

\section{OTH-005 PROSPECTIVE MULTI-SITE AUDIT OF ACUTE UPPER GASTROINTESTINAL BLEEDING DELIVERED ACROSS MULTIPLE REGIONS BY TRAINEE-LED NETWORKS}

\footnotetext{
${ }^{1}$ Richard James Michael Ingram* ${ }^{1}$ Andrew Alan Baxter, ${ }^{2}$ Keith Siau, ${ }^{2}$ Monika Maria Widlak, ${ }^{3}$ Katherine White, ${ }^{3}$ Kirsty Nixon. 'on behalf of GARNet (Gastroenterology Audit and Research Network, East Midlands) ${ }^{2}$ on behalf of WMRIG (West Midlands Research in Gastroenterology Group) ${ }^{3}$ on behalf of GasTRIN NoW (Gastroenterology Training Research and Improvement Network North West)
}

\subsection{6/gutjnl-2018-BSGAbstracts.527}

Introduction Trainee-led networks were developed to support improvements in patient care by delivering audit, quality improvement and research projects across multiple sites. The Midlands was the first UK region to establish gastroenterology trainee research networks: WMRIG in 2015 and the GARNet in 2016. North West trainees established GasTRIN NoW in 2017. Here, we report the first project in the UK to be delivered simultaneously by trainee physicians through multiple networks.

Methods We audited the care of patients with acute upper GI bleeding (AUGIB) against national quality standards (NICE QS38 and JAG GRS) and collected data on patient and process outcomes. Patients aged $\geq 16$ years admitted with suspected AUGIB who underwent an inpatient OGD were prospectively identified in a consecutive 30 day window from November 2017. Anonymised data and outcomes to $30 \mathrm{~d}$ were collected using common proformas and protocols.

Results 471 patients were identified across 30 sites (EM: 157, 8 sites, WM: 193, 12 sites, NW: 121, 10 sites). We report the population characteristics and selected audit and outcome measures for $455 / 471$ (97\%) with complete data available. The mean age was 65 years (SD 18.1, range 17-100) and $54 \%$ were male. $45 \%$ presented on weekdays $7 \mathrm{am}-7 \mathrm{pm}, 32 \%$ on weekdays outside these hours and $23 \%$ at weekends. The median time from presentation to OGD was 22.5 hour (IQR 12.7-43.9 hour), with 54\% performed within 24 hour (range at sites $22 \%-88 \% ;>50 \%$ : 18 sites, $>75 \%$ : 5 sites). Glasgow Blatchford scores were recorded pre-OGD in $47 \%$ (range at sites 8\%-100\%), with median score 7 (IQR 1-12; $\geq 8$ : $46 \%, \geq 12: 26 \%)$. Endoscopic findings were: $63 \%$ non-variceal, $11 \%$ variceal, $8 \%$ other, $18 \%$ no lesion identified. 116 patients required endotherapy (25\%; 80 non-variceal, 34 oesophageal and 4 gastric varices). A rebleed plan was documented in $36 \%$ (range at sites $0 \%-81 \%$ ). Rebleeding within $8 \mathrm{~d}$ occurred in 36 patients (7.9\%). 29 died (6.3\%) within $30 \mathrm{~d}$ of OGD. Median length of stay was $5 \mathrm{~d}$ (IQR 3-9 d).

Conclusions Collaboration between gastroenterology trainee-led research networks can successfully deliver projects across multiple sites and regions. This audit shows wide variations in practice. Local action plans are still needed to meet JAG quality measures. However, we propose to harness the enthusiasm of trainees to drive improvements in the quality of care received by patients with GI bleeding - regionally, through the BSG national QI agenda, and in partnership with patients via Core. We encourage our peers to establish their own trainee-led research networks.

\section{PTH-129 EVALUATION OF SIMULATION-BASED INDUCTION PROGRAMME TO ENHANCE ACQUISITION OF HANDLING SKILLS FOR UPPER GI ENDOSCOPY}

${ }^{1}$ Kate Edwards*, ${ }^{2}$ Keith Siau, ${ }^{1}$ Peter Neville, ${ }^{3}$ Jeff Turner, ${ }^{4}$ Amanda Beale, ${ }^{4}$ Susi Green, ${ }^{2}$ A Murugananthan, ${ }^{1}$ Neil Hawkes. ${ }^{1}$ Cwm Taf University Health Board, Merthyr Tydfil, UK ${ }^{2}$ Royal Wolverhampton NHS Trust, Wolverhampton, UK ${ }^{3}$ Cardiff and Vale UHB, Cardiff, UK ${ }^{4}$ Bristol Royal Infirmary, Bristol, UK ${ }^{5}$ Royal Sussex County Hospital, Sussex, UK

\subsection{6/gutjnl-2018-BSGAbstracts.528}

Introduction Endoscopic training pathways in the UK need to support efficient development of complex motor skills. Previous studies have demonstrated benefit from use of simulators in the early phase of training. However a target of a $25 \%$ improvement performance should be demonstrated to remain cost effective. The SPRINT induction programme aims to provide a structured sequence of training elements to optimise and accelerate the early phase of training in upper GI endoscopy. We hosted a Trideanery simulation training event using the Surgical Science EndoSIM.

Methods Baseline data on previous training experience was obtained from 20 trainees attending the SPRINT induction. Pre-course self-assessed competency scores in 12 upper GI handling skills domains were provided and mapped to the Joint Advisory Group on GI Endoscopy (JAG) DOPS assessment forms. Domain scores were given a rating from $0-10$ on a Likert scale $(0=$ not at all competent, $10=$ very competent $)$. Trainees completed a structured curriculum of simulator training and a series of small group teaching seminars on technical aspects of endoscopy and lesion recognition. At the end of the course trainees provided further self-assessed competency scores and rating scores for small group teaching sessions. Differences in the competency scores were determined by the Mann Whitney $U$ test with an alpha value of 0.01 . The proportion of trainees with less (0-25 procedures) and more experience (26-50 procedures) reporting greater than $25 \%$ domain score change was tested using the Chi-squared statistic.

Results Paired scores $(n=20)$ showed a significant increase across all 12 upper GI handling skills domains following the training intervention $(\mathrm{p}<0.01$, alpha $=0.01$, two-tailed). The proportion of trainees reporting $>25 \%$ difference in domain scores was greater for trainees with less experience compared to more experienced trainees $[\mathrm{p}<0.05$ for all domains]. All trainees rated good/excellent small group sessions in scope handling, lesion recognition, and endoscopic non-technical skills.

Conclusion This study demonstrates significant benefit from the EndoSIM simulator across all upper GI technical skills domains. Simulation training is best targeted in the early stages of endoscopy training ( $0-25$ cases), but there remains some benefit to more experienced trainees. Small group training provides additional value during the SPRINT induction. These findings further support inclusion of simulation as part of the JAG certification pathway for upper GI endoscopy. 